\title{
Reduction Efficacy in Aerosol Production using $0.2 \%$ Chlorhexidine Gluconate Mouthwash in Dental Waterlines: A Clinicomicrobiological Study
}

\author{
${ }^{1}$ Suresh K Kasagani, ${ }^{2}$ Aditya G Rao, ${ }^{3}$ Kafeel Ahmed, ${ }^{4}$ Ghousia Fatima, ${ }^{5}$ Roopali Tapashetti
}

\begin{abstract}
Introduction: Aerosol is a suspension of solid or liquid particles containing bacteria or viruses, suspended for at least a few seconds in a gas. The aerosol generated by an ultrasonic scaler contains microorganisms that can penetrate into the body through the respiratory system of dental surgeons and patients. The oral cavity harbors numerous bacteria and viruses from the respiratory tract, dental plaque, and oral fluids. Any dental procedure that has a potential to aerosolize saliva will cause airborne contamination with organisms.
\end{abstract}

Aim: To evaluate and compare the reduction efficacy in the levels of aerosol production at 1 foot, 5 feet, and 10 feet distance using $0.2 \%$ chlorhexidine gluconate mouthwash in dental waterlines.

Materials and methods: This single-center, randomized, twogroup parallel design study was conducted over a period of 20 days. Twenty patients with chronic periodontitis were randomly divided into two groups: Test group and control group. Both the group samples were subjected to ultrasonic scaling. In the test group, $0.2 \%$ chlorhexidine gluconate mouthwash was added in the water dispenser bottle, whereas in the control group, distilled water was used. Blood agar plates were kept at distances of 1 foot, 5 feet, and 10 feet away from the headrest of the dental chair. Blood agar plates were incubated at $37^{\circ} \mathrm{C}$ for 48 hours, and the total number of colony-forming units (CFUs) was counted and statistically analyzed.

Results: The results of this study revealed that the experimental group showed higher reduction efficacy for aerosol production at 1 foot, 5 feet, and 10 feet distance from the center of the headrest of the dental chair compared with the control group. The results of this study also revealed that the number of CFUs was statistically significant only at 1 foot distance $(p=0.009)$ from the center of the headrest of the dental chair and not at 5 feet $(p=0.122)$ and 10 feet $(p=0.507)$ distances for both the groups.

Conclusion: This present study shows that the patient's chest area at a distance of 1 foot from the center of the headrest of dental chair receives a greater number of microorganisms than that at distances of 5 feet and 10 feet from the headrest of the dental chair. This validates the use of chlorhexidine in the form of an irrigant in dental waterlines as an additional barrier

\footnotetext{
${ }^{1}$ Professor and Head, ${ }^{2}$ Senior Lecturer, ${ }^{3}$ Postgraduate Student ${ }^{4}$ Professor, ${ }^{5}$ Reader

${ }^{1-5}$ Department of Periodontics, Al Badar Rural Dental College \& Hospital, Kalaburagi, Karnataka, India

Corresponding Author: Kafeel Ahmed, Postgraduate Student Department of Periodontics, Al Badar Rural Dental College \& Hospital, Kalaburagi, Karnataka, India, e-mail: drkafeelsohar@ yahoo.in
}

to cross-contamination, minimizing the risk to team members and the patient.

Keywords: Aerosols, Colony-forming units, Ultrasonic scaling.

How to cite this article: Kasagani SK, Rao AG, Ahmed K, Fatima G, Tapashetti R. Reduction Efficacy in Aerosol Production using 0.2\% Chlorhexidine Gluconate Mouthwash in Dental Waterlines: A Clinicomicrobiological Study. J Health Sci Res 2016;7(1):1-5.

Source of support: Nil

Conflict of interest: None

\section{INTRODUCTION}

Periodontal disease is a microbe-induced chronic inflammatory condition affecting the periodontium. The oral cavity harbors numerous bacteria and viruses from the respiratory tract, dental plaque, and oral fluids. The combined action of water sprays, compressed air, organic particles, such as tissue and tooth dust, and organic fluids, such as blood and saliva from the site where the instrument is used produces airborne particles. ${ }^{1}$ Any dental procedure that has a potential to aerosolize saliva will cause airborne contamination with an organism. Dental hand pieces, ultrasonic scalers, air polishing devices, and air abrasion units produce airborne particles by the combined action of water sprays, compressed air, organic particles, such as tissue and tooth dust, organic fluids, such as blood and saliva. ${ }^{1}$ Aerosol is a suspension of solid or liquid particles containing bacteria or viruses, suspended (for at least a few seconds) in a gas with a particle size from 0.001 to $>100 \mathrm{~mm}^{2}$ The control and minimization of microorganisms contained in aerosol are of great importance to the health of dental personnel. Research shows that both the professional and the patient are exposed to high amounts of bacteria. According to the study conducted by Miller, ${ }^{2}$ aerosols generated from the patient's mouth contain up to 100,000 bacteria per cubic foot of air., The associations of these aerosols with the respiratory infections, ophthalmic and skin infections, tuberculosis, and hepatitis B have been reported in other studies. ${ }^{5}$ Several methods like using high-vacuum suction, patient positioning, use of rubber dams, and preprocedural antibacterial mouth rinses have been tried to reduce aerosol contamination in the dental office. ${ }^{6}$ 
The current literature suggests that having patients use an antimicrobial rinse before treatment may decrease microbial aerosols. ${ }^{7-10}$ Data from Fine and colleagues indicated that the irrigant used in dental waterlines with an antiseptic mouthwash reduced the microbial content of aerosols produced during treatment with an ultrasonic scaler. Chlorhexidine is considered as the "gold standard" of all the antimicrobial rinses, because of the broad-spectrum antibacterial activity and substantivity of 8 to 12 hours. ${ }^{11}$ However, none of the previous studies compared the reduction efficacy in aerosol production using any antibiotic mouthwash in dental waterlines. ${ }^{12-14}$ None of these studies correlated the distance and aerosol production.

\section{AIM}

The aim of the article was to evaluate and compare the reduction efficacy in aerosol production using $0.2 \%$ chlorhexidine gluconate mouthwash in dental waterlines. ${ }^{15}$ Hence, the objectives of this study were as follows: To evaluate the reduction efficacy in aerosol production using $0.2 \%$ chlorhexidine mouthwash in dental waterlines at 1 foot, 5 feet, and 10 feet from the center of the headrest of dental chair, and to evaluate the aerosol production using blood agar plates kept at the above-mentioned distance.

\section{MATERIALS AND METHODS}

Twenty subjects were selected randomly from the Department of Periodontics, Al Badar Rural Dental College \& Hospital, Kalaburagi, Karnataka, India. After ethical clearance, a written informed consent was obtained from all participants before the start of the study, and they were divided into two groups: Groups I and II.

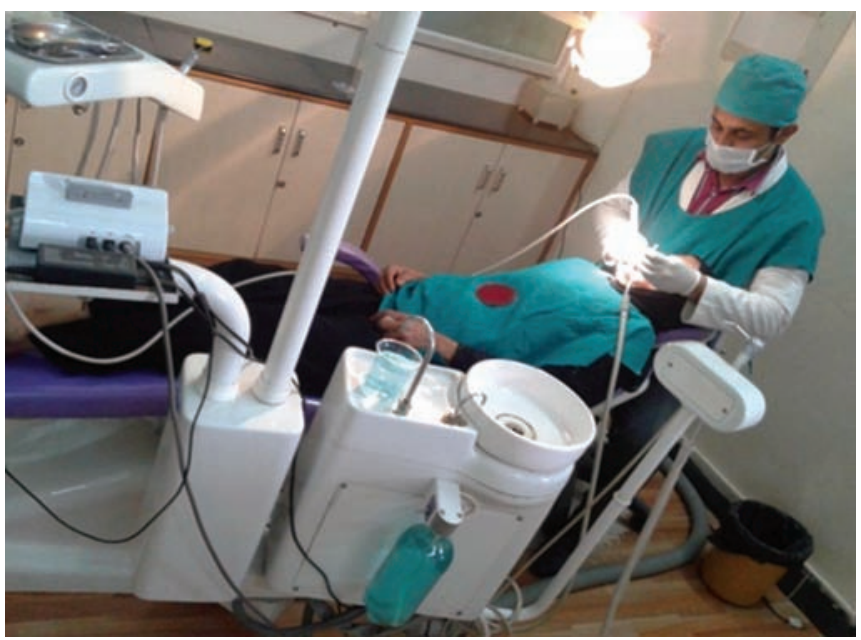

Fig. 1: Test group: $0.2 \%$ chlorhexidine mouthwash added to the dilution of 1:10 ratio in dental waterlines
Group I included 10 subjects with chronic periodontitis undergoing ultrasonic scaling with distilled water. Group II included 10 subjects with chronic periodontitis undergoing ultrasonic scaling with antimicrobial irrigant $(10 \mathrm{~mL}$ of undiluted $0.2 \%$ chlorhexidine) used in the dental waterlines.

\section{INCLUSION CRITERIA}

Inclusion criteria included chronic periodontitis patients between the ages of 20 and 60 years, with minimum 20 teeth showing more than $30 \%$ of sites with clinical attachment loss of more than $4 \mathrm{~mm}$ (Table 1).

\section{EXCLUSION CRITERIA}

The exclusion criteria included patients with any systemic disease, subjects taking antibiotic or any other drugs within the past 3 months, pregnant and lactating women, medically compromised patients, and smokers.

This single-center, placebo-controlled, randomized, two-group, parallel design study was conducted over a period of 20 days. Blood agar plates were used to collect airborne microorganisms. Johnston et $\mathrm{a}^{16}$ proved that blood agar plates are a valid medium for culturing airborne bacteria. Three standardized locations were chosen to be evaluated for each treatment group, viz., 1 foot, 5 feet, and 10 feet from the center of the head rest of dental chair. The same closed location was used for all treatment procedures. Before each appointment, a staff cleaned, disinfected, and fumigated all surfaces using 37 to $41 \%$ formalin. Twenty chronic periodontitis patients were recruited in chronological order and were randomly allocated to one of two groups based on the irrigant used in dental waterlines. In the test group, $0.2 \%$ chlorhexidine was diluted in 1:10 ratio in dental waterlines (Fig. 1), whereas in the control group, distilled water was used in dental waterlines (Fig. 2).

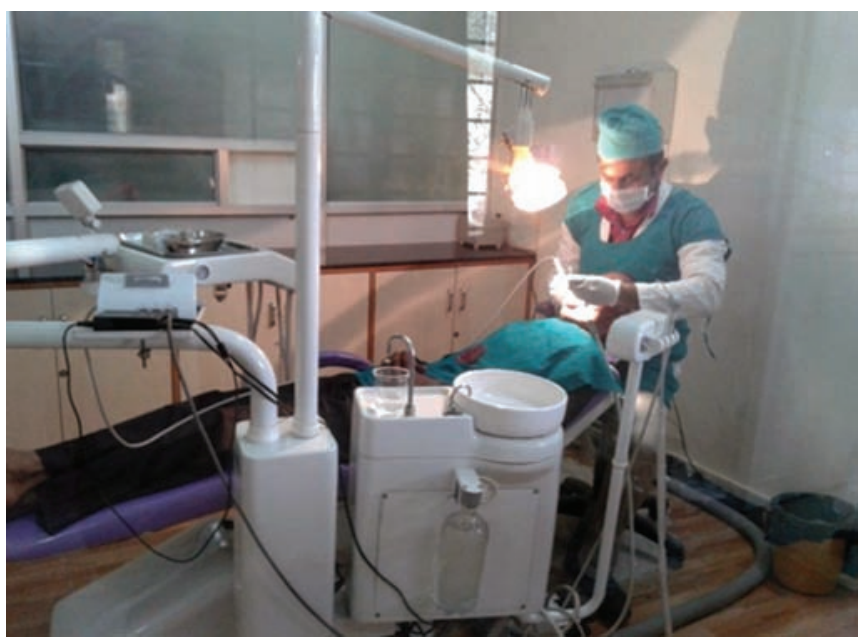

Fig. 2: Control group: Distilled water used in dental waterlines 
Each treatment session consisted of 30 minutes of ultrasonic scaling. During the treatment and for 30 minutes after the treatment, three coded blood agar plates were left uncovered at predesignated sites to collect samples of any aerosolized bacteria at 1 foot, 5 feet, and 10 feet distances from the center of the headrest of the dental chair. After collecting the samples, the blood agar plates were incubated at $37^{\circ} \mathrm{C}$ for 48 hours. The number of colony-forming units (CFUs) that grow on each plate was counted at the Microbiology Department of Al Badar Rural Dental College \& Hospital, Kalaburagi, Karnataka, India.

\section{Statistical Analysis}

All the samples collected were subjected to statistical analysis. Comparison of the two groups was done using unpaired t-test. Intragroup comparison of colonies \{mean [standard deviation (SD)]\} collected from different distances in the control and test groups was carried out using the analysis of variance (ANOVA) test. Intergroup comparison of colonies [mean (SD)] collected from different distances in the control and test groups was also performed using the ANOVA test.

\section{RESULTS}

This study shows that the patient's chest area, i.e., 1 foot distance receives a greater number of microorganisms than that of 5 feet and 10 feet distances from the center of the headrest of the dental chair.

The number of CFUs was highest at the 1 foot distance of the patient's chest area and lowest at the 10 feet distance from the center of the headrest of the dental chair (Figs 3 to 5). The number of CFUs formed on blood agar plates was less in the test group (chlorhexidine irrigant used in dental waterlines) than in the control group where mouthwash was not used. Thus, chlorhexidine was proven to be more effective in reducing the number of CFUs on agar plates compared with water when used as an irrigant in dental waterlines for oral prophylaxis.
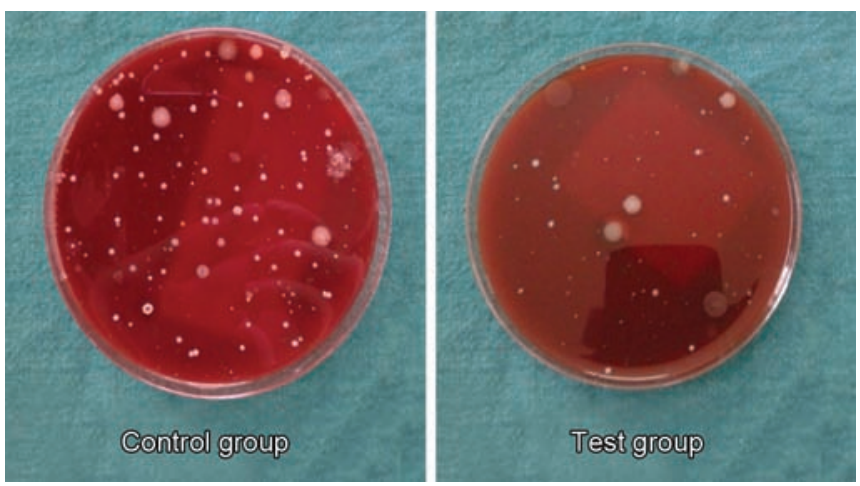

Fig. 4: Five feet from the center of the headrest of the dental chair
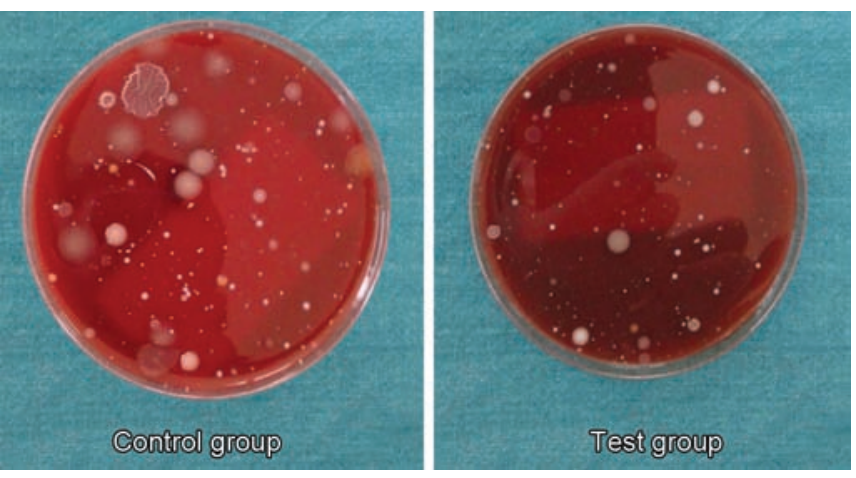

Fig. 3: One foot from the center of the headrest of the dental chair

In Table 2, the CFUs were compared at different distances in the control group. It shows the maximum number of CFUs at 1 foot distance (86.30), compared with 5 feet (70.50) and 10 feet (45.90) distances. This difference was highly significant with a p-value of $<0.001$.

In Table 3, the CFUs were compared at different distances in the chlorhexidine group. It reveals the maximum number of CFUs at 1 foot distance (65.30) compared with 5 feet (55.80) and 10 feet (42.90) distances. This difference was also highly significant, with a p-value of $<0.001$.

In Table 4, the number of CFUs was more in the control group (86.30) compared with the chlorhexidine group (65.30). This difference was statistically significant, with a p-value of 0.009 .

The CFUs were more in the control group (70.50) compared with the chlorhexidine group (55.80). However, this difference was not significant, with a p-value of 0.122 (Table 5).

This calculation showed no significant difference with a p-value of 0.507 . However, there were less number of CFUs in the chlorhexidine group compared with the control group (Table 6).

Overall, the results clearly suggest that $0.2 \%$ chlorhexidine used as an irrgant in dental waterlines is a more effective primary measure than control group in reducing aerosol cross-contamination during use of ultrasonic scaling in the practice of dentistry.
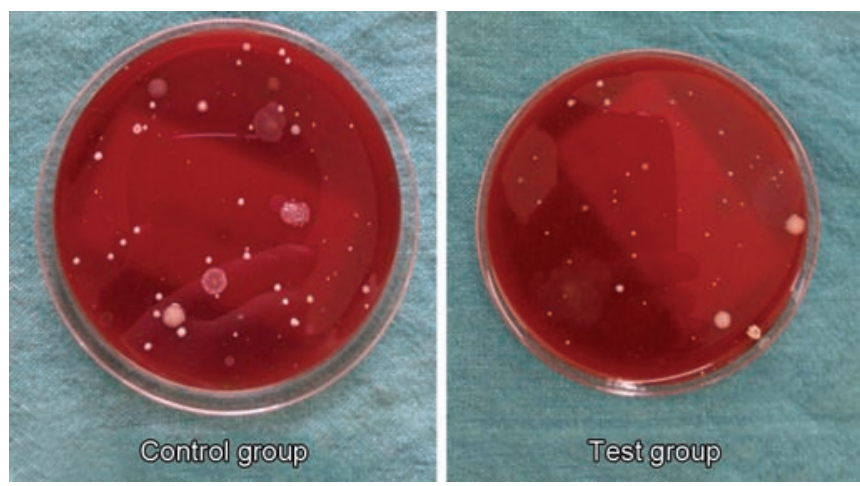

Fig. 5: Ten feet from the center of the headrest of the dental chair 
Table 1: Comparison of age among both the groups using unpaired t-test

\begin{tabular}{lll}
\hline Groups & Number of samples & Mean (SD) \\
\hline Control & 10 & $41.10(12.1)$ \\
Chlorhexidine & 10 & $46.50(11.8)$ \\
t-value & - & 1.011 \\
p-value & - & 0.325 \\
\hline
\end{tabular}

Table 3: Comparison of colonies collected from different distances in the chlorhexidine group using ANOVA test

\begin{tabular}{lll}
\hline Distance & Number of samples & Mean (SD) \\
\hline 1 foot & 10 & $65.30(8.8)$ \\
5 feet & 10 & $55.80(14.4)$ \\
10 feet & 10 & $42.90(11.8)$ \\
f-value & - & 8.970 \\
p-value & - & $<0.001^{* *}$ \\
\hline
\end{tabular}

*Significant when $p<0.05$; **highly significant when $p<0.001$

Table 5: Comparison of colonies collected at a distance of 5 feet among both the groups using unpaired t-test

\begin{tabular}{lll}
\hline Groups & Number of samples & Mean (SD) \\
\hline Control & 10 & $70.50(24.8)$ \\
Chlorhexidine & 10 & $55.80(14.4)$ \\
t-value & - & 1.622 \\
p-value & - & 0.122 \\
\hline
\end{tabular}

\section{DISCUSSION}

Miller ${ }^{2}$ found that aerosols generated from a patient's mouth contained up to a million bacteria per cubic foot of air. Other studies have reported the association of these aerosols with respiratory infections, ${ }^{2}$ ophthalmic and skin infections, ${ }^{2}$ tuberculosis, and hepatitis B., ${ }^{4,6}$ This clinicomicrobiological study was conducted on 20 patients with chronic periodontitis. Microbiological evaluation was done using culture tests as it happens to be the gold standard method for its evaluation. ${ }^{9}$ The results of this study revealed that the experimental group showed higher reduction efficacy for aerosol production at 1 foot, 5 feet, and 10 feet distances from the center of the headrest of the dental chair compared with the control group. Higher reduction efficacy in aerosol production by $0.2 \%$ chlorhexidine could be attributed to its antimicrobial property. ${ }^{8}$ The results of this study are in accordance with the study done by Gunjan et al where the authors used chlorhexidine for preprocedural mouth rinse. The results of this study also revealed that the number of CFUs was statistically significant only at 1 foot distance $(p=0.009)$ from the center of the headrest of the dental chair and not at 5 feet $(p=0.122)$ and 10 feet $(p=0.507)$ distances for both the groups. Thus, within the limits of this study, it could be hypothesized that aerosol production is inversely proportional to the distance from the ultrasonic scaling device. ${ }^{11}$ Studies have also shown that ultrasonic
Table 2: Comparison of colonies collected from different distances in the control group using ANOVA test

\begin{tabular}{lll}
\hline Distance & Number of samples & Mean (SD) \\
\hline 1 foot & 10 & $86.30(20.8)$ \\
5 feet & 10 & $70.50(24.8)$ \\
10 feet & 10 & $45.90(7.6)$ \\
f-value & - & 11.271 \\
p-value & - & $<0.001^{\star *}$ \\
\hline
\end{tabular}

*Significant when $p<0.05$; **highly significant when $p<0.001$

Table 4: Comparison of colonies collected at a distance of 1 foot among both the groups using unpaired t-test

\begin{tabular}{lll}
\hline Group & Number of samples & Mean $(S D)$ \\
\hline Control & 10 & $86.30(20.8)$ \\
Chlorhexidine & 10 & $65.30(8.8)$ \\
t-value & - & 2.945 \\
p-value & - & $0.009^{*}$ \\
\hline
\end{tabular}

Table 6: Comparison of colonies collected at a distance of 10 feet among both the groups using unpaired t-test

\begin{tabular}{lll}
\hline Groups & Number of samples & Mean $(\mathrm{SD})$ \\
\hline Control & 10 & $45.90(7.6)$ \\
Chlorhexidine & 10 & $42.90(11.8)$ \\
t-value & - & 0.677 \\
p-value & - & 0.507 \\
\hline
\end{tabular}

scaling in conjunction with various plaque control agents (chlorhexidine) used as an irrigant in dental waterlines has been found to be more effective in reducing bacterial loads compared with distilled water. ${ }^{15-17}$ The limitations of this study should be considered in interpreting these results. The CFUs counted here are the values that represent only aerobic bacteria capable of growth on blood agar plates; viruses, anaerobia bacteria, and organisms requiring specialized media were not cultured in this study. ${ }^{16}$ This study shows that the patient's chest area receives a greater number of microorganisms at a distance of 1 foot than that of the dental professional, followed by that at 5 feet and 10 feet distances from the center of the headrest of the dental chair. This study revealed that both the dentist and the patient were exposed to high amounts of bacteria due to aerosols produced by ultrasonic scaling. The highest number of colonies was seen on the plates positioned on the patient's chest area. The larger salivary droplets generated during dental procedures settle down rapidly from the air with heavy contamination of a plate placed at the patient's chest area. When $10 \mathrm{~mL}$ of undiluted $0.2 \%$ chlorhexidine was used as an irrigant in dental waterlines, fewer CFUs developed than the ultrasonic scaling done without the use of mouthwash. ${ }^{11-13}$ The results of this study clearly indicate that $0.2 \%$ chlorhexidine gluconate used as an irrigant in dental waterlines was significantly effective in reducing 
the aerosol contamination during the use of ultrasonic scaling in dental practice. Various other studies support the results of this study demonstrating the excellent antimicrobial effects of $0.2 \%$ chlorhexidine used as an irrigant in dental waterlines in aerosol reduction.

\section{CONCLUSION}

This study shows that the patient's chest area at a distance of 1 foot from the center of the headrest of the dental chair receives a greater number of microorganisms than at distances of 5 feet and 10 feet from the headrest of the dental chair. This reinforces the importance of using personal protective equipment, such as eye and face shields, head cap, mask, gloves, and gowns/white coats. This validates the use of chlorhexidine in the form of irrigant in dental waterlines as an additional barrier to crosscontamination, minimizing the risk to team members and the patient.

\section{REFERENCES}

1. Acharya S, Priya H, Purohit B, Bhat M. Aerosol contamination in a rural university dental clinic in south India. Int J Infect Control 2010;6(1):2-7.

2. Miller RL. Generation of airborne infection by high speed dental equipment. J Am Soc Prev Dent 1976 May-Jun;6(3):14-17.

3. Federal Register. Department of Labor. Occupational Safety and Health Administration. Occupational exposure to bloodborne pathogens; final rule. 29 CFR part 1910.1030. Part II. 1991;56(235):64175-64182.

4. Shaw AB. Tuberculosis in medical and dental students; a study at Guy's hospital. Lancet 1952 Aug;2(6731):400-404.

5. Hinds WC. Aerosol technology: properties, behavior, and measurement of airborne particles. New York: Wiley; 1982. p. 6.
6. Goldman HS, Hartman KS. Infectious diseases. Their disease, our unease: infectious diseases and dental practice. Va Dent J 1986 Apr-Jun;63(2):10-19.

7. Worrall SF, Knibbs PJ, Glenwright HD. Methods of reducing bacterial contamination of the atmosphere arising from use of an air-polisher. Br Dent J 1987 Aug;163(4):118-119.

8. Glenwright HD, Knibbs PJ, Burdon DW. Atmospheric contamination during use of an air polisher. Br Dent J 1985 Nov;159(9):294-297.

9. Weaks LM, Lescher NB, Barnes CM, Holroyd SV. Clinical evaluation of the Prophy-Jet as an instrument for routine removal of tooth stain and plaque. J Periodontol 1984 Aug;55(8): 486-488.

10. Orton GS. Clinical use of an air-powder abrasive system. Dent Hyg (Chic) 1987 Nov;61(11):513-518.

11. Lyle D. The role of pharmacotherapeutics in the reduction of plaque and gingivitis. J Pract Hyg 2000;9(6):46-50.

12. Logothetis DD, Martinez-Welles JM. Reducing bacterial aerosol contamination with a chlorhexidine gluconate prerinse. J Am Dent Assoc 1995 Dec;126(12):1634-1639.

13. Son WK, Shin SY, Kye SB, Yang SM. The effect of chlorhexidine on reduction of viable organisms in aerosol produced by ultrasonic scaler. J Korean Acad Periodontol 2009 Sep;39(3):303-310.

14. Reddy S, Prasad MG, KaulS, Satish K, Kakarala S, BhowmikN. Efficacy of $0.2 \%$ tempered chlorhexidine as a pre-procedural mouth rinse: a clinical study. J Indian Soc Periodontol 2012 Apr;16(2):213-217.

15. Van der Weijden GA, Timmer CJ, Timmerman MF, Reijerse E, Mantel MS, van der Velden U. The effect of herbal extracts in an experimental mouthrinse on established plaque and gingivitis. J Clin Periodontol 1998 May;25(5):399-403.

16. Johnston JR, Butchart AM, Kgamphe SJ. A comparison of sampling methods for airborne bacteria. Environ Res 1978 Jul;16(1-3):279-284.

17. Serfaty R, Itic J. Comparative clinical trial with natural herbal mouthwash versus chlorhexidine in gingivitis. J Clin Dent 1988 Summer;1(Suppl A):A34-A37. 